Table I. The Flavones of Sixteen

\section{Identification of Flavones in Sixteen Compositae Species}

\author{
Makoto KaneTA, Hiroshi HikICHI, \\ Seiichi ENDO and Noboru SugIYAMA* \\ Fukushima Technical College, Iwaki-shi, Fukushima \\ * Department of Chentistry, Tokyo Kyoiku \\ University, Otsuka, Tokyo \\ Received August 16, 1977
}

In the previous papers, ${ }^{1)}$ we described the investigation for flavones of the genus Gramineae. The present paper describes the identification of flavones in sixteen Compositae species by paper chromatography and ultraviolet spectroscopy. In this work, the presence of apigenin (1), apigenin 7-O-glucoside (2), apigenin 7-O-rhamnoglucoside (3), acacetin 7-O-glucoside (4), acacetin 7-O-rhamnoglucoside (5), luteolin (6), luteolin 7-O-glucoside (7), luteolin 7-O-rhamnoglucoside (8), quercetin 3-O-galactoside (9), quercitrin (10), isorhamnetin 3-O-galactoside (11) has been demonstrated in the plants. The methods used for identification are outlined below. Methanol extracts of fresh plants were separated by two-dimensional paper chromatography, and the developed chromatograms were eluted separately with methanol. The ultraviolet spectral characteristics of the eluates were compared with the data reported for known compounds for the identification of the flavones in the plant. For the identification of glycoside, the eluate from chromatogram was subjected to acid hydrolysis to yield aglycone and sugar which was identified by paper chromatography and ultraviolet spectroscopy. The identified flavones are shown in Table $I$ in which the compounds marked with a) have not yet been reported in those plants. The ultraviolet spectral data and the data of acid hydrolysis shown in Table II agreed with those reported for known compounds. ${ }^{\text {2) }}$

\section{EXPERIMENTAL}

Fresh stems and leaves were extracted with methanol for $40 \sim 50 \mathrm{hr}$. Evaporation of methanol under reduced pressure yielded a sticky residue, which was dissolved in a small portion of methanol. Spots of the extracts on Whatman $3 \mathrm{MM}$ chromatographic paper $(46 \times$ $57 \mathrm{~cm}$ ) was developed by descending two-dimensional chromatography using TBA (tertiary butanol: glacial acetic acid: water $=3: 1: 1 \mathrm{v} / \mathrm{v}$ ) as the first solvent and HOAc (glacial acetic acid: water $=15: 85 \mathrm{v} / \mathrm{v}$ ) as the second solvent. All of the detected spots were cut off from the paper and eluted separately with methanol.
Compositae SPECIES

\begin{tabular}{|c|c|}
\hline Species & Compounds identified \\
\hline Artemisia jo & $\begin{array}{l}\text { Apigenin } 7-O \text {-glucoside } \\
\text { Luteolin } 7-O \text {-glucoside } \\
\text { a) }\end{array}$ \\
\hline Aster leiophyl & Apigenin 7-O-glucoside \\
\hline Chrysanthemum morifolium & Apigenin 7-O-glucoside \\
\hline Ramat & $\begin{array}{l}\text { Luteolin 7-O-glucoside } \\
\text { Acacetin 7-O-rhamno- } \\
\text { glucoside }\end{array}$ \\
\hline $\begin{array}{l}\text { Cirsium nipponicum } \\
\text { (Maxim.) }\end{array}$ & Apigenin 7-O-glucoside $\left.{ }^{\alpha}\right)$ \\
\hline Makino & Lut \\
\hline Cirsium purpuratum & Apigenin $^{a}$ ) \\
\hline
\end{tabular}

Apigenin 7-O-glucoside $\mathrm{e}^{a}$ Acacetin 7-O-glucoside ${ }^{a}$ Quercitrin $^{a)}$

Cosmos bipinnatus Cav

Apigenin 7-O-glucoside Quercitrin $^{a}$ )

Dahlia pinnata Cav

Erigeron acris $\mathrm{L}$

Erigeron annuus (L.) Pers

Apigenin 7-O-glucoside Luteolin 7-O-glucoside ${ }^{a>}$

Apigenin 7-O-rhamnoglucoside $^{a>}$

Quercitrin $^{a}$ )

\section{Hypochoeris radicata $\mathrm{L}$ \\ Kalimeris pinnatifida \\ (Maxim.) Kitam}

Lactuca indica $\mathrm{L}$

Luteolin 7-O-glucoside ${ }^{\text {3 }}$

Isorhamnetin 3-Ogalactoside $^{a)}$

Apigenin 7-O-glucoside ${ }^{a}$

Apigenin 7-O-glucoside ${ }^{a}$ )

Luteolin 7-O-glucoside ${ }^{a)}$

Quercetin 3-O-galactoside $^{a)}$

Sonchus asper (L.) Vill

Apigenin 7-O-rhamnoglucoside $^{\text {3 }}$ )

Luteolin 7-O-glucoside ${ }^{a}$ )

Luteolin 7-O-rhamnoglucoside $^{a}$ )

Sonchus oleraceus L

Luteolin $^{a}$ )

Luteolin 7-O-glucoside ${ }^{a)}$

Apigenin 7-O-glucoside $\mathrm{e}^{a}$

Taraxacum platycarpum

Apigenin 7-O-glucoside ${ }^{a}$

Luteolin 7-O-glucoside $\mathrm{e}^{a}$

Dahlst

Youngia denticulata

Luteolin 7-O-glucoside ${ }^{a)}$

(Houtt.) Kitam

a) First identified by our work.

In this way, chromatograms of about 30 sheets of chromatographic paper in each plant have been developed. The eluate thus obtained was used for spectral analyses. The ultraviolet spectra were measured in methanol, sodium methylate, methanolic aluminum chloride, methanolic aluminum chloride-hydrochloric acid, methanolic sodium acetate, and methanolic sodium acetate-boric acid solution by an application of the method of Mabry et al. ${ }^{2}$ Substance which was suspected to be a glycoside was subjected to acid hydrolysis. The combined eluate from about 30 chromatograms was concentrated almost to dryness and was 
Table II. IDentification of Flavones

\begin{tabular}{|c|c|c|c|c|c|c|}
\hline \multirow[t]{2}{*}{ Substance } & \multicolumn{4}{|c|}{ UV spectral data $\max , \mathrm{nm}$} & \multirow{2}{*}{$\begin{array}{l}\text { Products by } \\
\text { acid hydrolysis }\end{array}$} & \multirow[t]{2}{*}{ Identity } \\
\hline & $\mathrm{MeOH}$ & $\mathrm{NaOMe}$ & $\mathrm{AlCl}_{3}$ & $\mathrm{AlCl}_{B} / \mathrm{HCl}$ & & \\
\hline \multirow[t]{4}{*}{ Sost. 1} & 267 & 275 & 276 & 276 & & Apigenin \\
\hline & 336 & 325 & 300 & 299 & & \\
\hline & & 393 & 348 & 340 & & \\
\hline & & & 385 & 380 & & \\
\hline \multirow[t]{4}{*}{ Sbst. 2} & 268 & 270 & 276 & 277 & Apigenin & Apigenin 7-0- \\
\hline & 333 & $298 \mathrm{sh}$ & 300 & 299 & Glucose & glucoside \\
\hline & & 385 & 348 & 340 & & \\
\hline & & & 386 & 382 & & \\
\hline \multirow[t]{4}{*}{ Sbst. 3} & 267 & 245 & 275 & 276 & Apigenin & Apigenin 7-O. \\
\hline & 333 & 267 & 298 & 299 & Glucose & rhamnoglucoside \\
\hline & & $300 \mathrm{sh}$ & 346 & 341 & Rhamnose & \\
\hline & & 385 & 382 & 380 & & \\
\hline \multirow[t]{4}{*}{ Sbst. 4} & 268 & 285 & 277 & 278 & Acacetin & Acacetin $7-0$ \\
\hline & 324 & 359 & 300 & 300 & Glucose & glucoside \\
\hline & & & 345 & 338 & & \\
\hline & & & 384 & 381 & & \\
\hline \multirow[t]{4}{*}{ Sbst. 5} & 268 & 286 & 277 & 278 & Acacetin & Acacetin 7-O \\
\hline & 324 & 358 & 300 & 300 & Glucose & rhamnoglycoside \\
\hline & & & 345 & 340 & Rhamnose & \\
\hline & & & 384 & 382 & & \\
\hline \multirow[t]{5}{*}{ Sbst. 6} & 253 & 266 & 273 & 263 & & Luteolin \\
\hline & $267 \mathrm{sh}$ & $329 \mathrm{sh}$ & $300 \mathrm{sh}$ & 275 & & \\
\hline & $290 \mathrm{sh}$ & 400 & 328 & 295 & & \\
\hline & 348 & & 426 & 355 & & \\
\hline & & & & 385 & & \\
\hline \multirow[t]{4}{*}{ Sbst. 7} & 255 & 263 & 274 & 274 & Luteolin & Luteolin 7-O- \\
\hline & $267 \mathrm{sh}$ & $300 \mathrm{sh}$ & $298 \mathrm{sh}$ & $294 \mathrm{sh}$ & Glucose & glucoside \\
\hline & 348 & 394 & $330 \mathrm{sh}$ & 357 & & \\
\hline & & & 432 & 387 & & \\
\hline \multirow[t]{4}{*}{ Sbst. 8} & 255 & 263 & 272 & 272 & Luteolin & Luteolin 7-0- \\
\hline & $265 \mathrm{sh}$ & $300 \mathrm{sh}$ & $296 \mathrm{sh}$ & 295 & Glucose & rhamnoglucoside \\
\hline & 349 & 394 & $330 \mathrm{sh}$ & 359 & Rhamnose & \\
\hline & & & 432 & 388 & & \\
\hline \multirow[t]{4}{*}{ Sbst. 9} & 257 & 272 & 275 & 268 & Quercetin & Quercetin 3-O- \\
\hline & 269 & 327 & $305 \mathrm{sh}$ & $300 \mathrm{sh}$ & Galactose & galactoside \\
\hline & $300 \mathrm{sh}$ & 408 & $330 \mathrm{sh}$ & $365 \mathrm{sh}$ & & \\
\hline & 360 & & 436 & 403 & & \\
\hline \multirow[t]{4}{*}{ Sbst. 10} & 256 & 270 & 275 & 272 & Quercetin & Quercitrin \\
\hline & $265 \mathrm{sh}$ & 326 & $303 \mathrm{sh}$ & $303 \mathrm{sh}$ & Rhamnose & \\
\hline & $300 \mathrm{sh}$ & 395 & 333 & 353 & & \\
\hline & 350 & & 430 & 400 & & \\
\hline \multirow{4}{*}{ Sbst. 11} & 255 & 272 & 270 & 267 & Isorhamnetin & Isorhamnetin 3-O- \\
\hline & $267 \mathrm{sh}$ & 327 & $300 \mathrm{sh}$ & $300 \mathrm{sh}$ & Galactose & galactoside \\
\hline & 300 sh & 415 & $365 \mathrm{sh}$ & 357 & & \\
\hline & 357 & & 407 & 403 & & \\
\hline
\end{tabular}

mixted with $6 \%$ aqueous hydrochloric acid $(3 \sim 5 \mathrm{ml})$ using a minimum of methanol to effect complete solution. The solution was heated on a steam bath for $2 \sim$ $3 \mathrm{hr}$ and then cooled and extracted with ether. Evaporation of the aqueous layer yielded sugars which were identified by paper chromatography in two solvent systems; $n$-butanol: acetic acid: water $=4: 1$ : $5 \mathrm{v} / \mathrm{v}$, and ethyl acetate: pyridine: water $=2: 1: 2 \mathrm{v} / \mathrm{v}$. Authentic samples of sugar were chromatographed alongside as check. The ether layer, after drying over sodium sulfate yielded aglycones which were identified by ultraviolet spectroscopy by the procedure de- 
scribed above.

\section{REFERENCES}

1) M. Kaneta and N. Sugiyama, Bull. Chem. Soc. Jpn., 42, 2084 (1969); idem, ibid., 45, 528 (1972); idem, J. Chem. Soc., 1971, 1982; idem, Agric.
Biol. Chem., 37, 2663 (1973).

2) "The Systematic Identification of Flavonoids," ed. by T. J. Mabry, K. R. Markham and M. B. Thomas, Springer-Verlag, New York, 1970, p. $81,82,83,91,95,96,97,128,129,139,27$, and pp. $35 \sim 38$. 